\title{
Análisis del Efecto Día de Semana en los principales mercados accionarios latinoamericanos: una aproximación mediante el criterio de Dominancia Estocástica*
}

Analysis of Day of the Week Effect in the main Latin-American stock markets: an approximation through the Stochastic Dominance Criterion

\author{
WERNER KRISTJANPOLLER** \\ Roberto E. MuÑOZ***
}

\begin{abstract}
Resumen
En este artículo estudiamos la presencia de anomalías de calendario en los principales mercados accionarios latinoamericanos en el período comprendido entre 1993 y 2007. La literatura ha mostrado que la detección de tales efectos puede depender de los supuestos de distribución de errores en el análisis econométrico (Baker et al., 2008) y que la existencia misma de ellos podría deberse a un problema de data snooping (Sullivan et al., 2001). En respuesta a estos problemas, Cho et al. (2007) introdujeron un test no-paramétrico robusto, el que fue adoptado en este artículo. Los resultados muestran que las anomalías de calendario: Efectos Lunes y Fin de Semana están presentes en las principales bolsas latinoamericanas y son estadísticamente significativas.
\end{abstract}

Palabras clave: Efecto Día de Semana, Dominancia estocástica, Mercados emergentes.

Clasificación JEL: F3, F4, G1.

\begin{abstract}
In this paper we study the presence of calendar anomalies in the main LatinAmerican stock markets, for the 1993 to 2007 period. The literature has shown that the detection of those effects may depend on error distribution assumptions (Baker et al., 2008), and that their existence could be due to a problem of data snooping (Sullivan et al., 2001). In response to these problems, Cho et al. (2007)

* Los autores desean agradecer los comentarios del Editor y un referee anónimo que permitieron introducir las comparaciones con el trabajo de Sullivan et al. (2001), así como realizar los análisis de sensibilidad y de significancia económica de los resultados incorporados en esta versión.

** Departamento de Industrias, Universidad Técnica Federico Santa María, Santiago, Chile, E-mail: werner.kristjanpoller@usm.cl

*** Departamento de Industrias, Universidad Técnica Federico Santa María, Santiago, Chile, E-mail: roberto.munoz@usm.cl
\end{abstract}


introduced a robust non-parametric test, which was adopted in this paper. Results show that calendar anomalies: Monday and Weekend Effects are present in the main Latin-American stock markets, and they are statistically significant.

Keywords: Day of the Week Effect, Stochastic Dominance, Emerging Markets. JEL Classification: F3, F4, G1.

\section{INTRODUCCIÓN}

Las anomalías de calendario, entre ellas el Efecto Día de Semana, han representado un enigma para los mercados financieros y una aparente contradicción para la teoría de mercados eficientes.

El Efecto Día de Semana en los mercados accionarios consiste en que la distribución de los retornos de un día es diferente a la de los otros días. Específicamente se pueden destacar el Efecto Lunes y el Efecto Fin de Semana. El Efecto Lunes se caracteriza porque el día lunes tendría una rentabilidad menor que los demás días y frecuentemente una mayor volatilidad. Por el contrario, el Efecto Fin de Semana tiene como característica que el día viernes presentaría un mayor retorno que el resto de los días de la semana ${ }^{1}$.

La vigencia de estas anomalías, sin embargo, ha sido fuertemente cuestionada en la última década. Por una parte, el mejor acceso a información bursátil y una mayor profundidad de los mercados financieros implicarían que toda posibilidad de arbitraje debiera tender a desaparecer ${ }^{2}$. Por otra parte, trabajos académicos recientes han cuestionado la existencia histórica de estos efectos.

Primero, el trabajo de Sullivan et al. (2001) atribuye la detección de estos efectos a un problema de data snooping. Segundo, un artículo reciente de Baker et al. (2008) mostró que, en las aproximaciones tradicionales, las conclusiones respecto a la presencia o ausencia del fenómeno dependen de los supuestos adoptados sobre la distribución de los errores.

En respuesta a los cuestionamientos anteriores, Cho et al. (2007) introducen un nuevo test no paramétrico, robusto a los cuestionamientos mencionados, para estudiar la presencia de dominancia estocástica de los retornos de unos días por sobre otros. Si bien los resultados de este test podrían haber confirmado la ausencia de tales efectos, los autores muestran que ellos persisten en la mayoría de los índices analizados ${ }^{3}$. El tema, por tanto, ha adquirido nuevamente gran relevancia, especialmente en Latinoamérica, donde los mercados financieros han logrado mayor profundidad y facilidad de acceso a transacciones.

Este artículo es, según sabemos, el primero que aplica la aproximación de Cho et al. (2007) a las principales bolsas latinoamericanas. Los resultados

1 Para revisar distintas aproximaciones metodológicas ver, por ejemplo, Kiymaz y Berument (2003), Brusa y Liu (2004) y Charles (2010).

2 Steeley (2001) encuentra que, en efecto, el fenómeno ha tendido a desaparecer en Inglaterra.

3 El Efecto Lunes es, sin embargo, más fuerte en el caso del Nasdaq y Russell 2000 que en el Dow Jones Industrial Average (DJIA) y S\&P 500, que son los índices analizados. 
muestran la persistencia de las anomalías de calendario en Latinoamérica. Las implicancias para los tomadores de decisiones son evidentes; por ejemplo, para la definición del día de compra o venta de activos es información valiosa saber que un día viernes domina a un lunes en rentabilidad.

\section{Efecto Día de Semana}

El Efecto Día de Semana fue descrito por primera vez en un artículo de Fields (1931), donde al analizar el índice Dow Jones con datos diarios en el período 1915-1930 encontró una significativa diferencia entre la rentabilidad media de los días lunes y la de los días viernes. El tema mantuvo su vigencia por décadas, como muestran los estudios de Fama (1965) y Cross (1973), quienes encontraron evidencia de que los días lunes se caracterizan por un bajo retorno y alta volatilidad en comparación con los demás días de la semana.

French (1980) analizó el comportamiento del Índice S\&P 500 en el período 1953-1977. Para estudiar la presencia del efecto, considera dos hipótesis alternativas de análisis de retornos: hipótesis del tiempo calendario, la cual incorpora el tiempo de no transacción en el día hábil siguiente, e hipótesis del tiempo de transacción, en la cual los días festivos no son tomados en cuenta en la base temporal. Los resultados obtenidos fueron una rentabilidad de los días lunes bajo el promedio de todo el período analizado, independientemente de la hipótesis adoptada. Más aún, analizando los retornos de cada día en subperíodos de cinco años, la rentabilidad promedio de los días lunes fue negativa.

Desde la década del 80 hasta la actualidad, numerosos trabajos se han realizado para estudiar la presencia o ausencia del fenómeno Efecto Día de Semana. Dentro de estos estudios se pueden mencionar los realizados por Gibbons \& Hess (1981), Lakonishok \& Levi (1982) o Rogalski (1984). La gran mayoría de ellos utilizan como metodología la regresión lineal sobre los retornos diarios utilizando dummies por día. Gibbons \& Hess (1981) encuentran evidencia que los retornos del día lunes son menores en promedio que los retornos de los otros días e incluso su promedio es negativo (lo que permitiría explotar la anomalía). Lakonishok \& Levi (1982) abordan el Efecto Día de Semana buscando una explicación parcial del fenómeno asociada a características de las transacciones del mercado, mientras que Rogalski (1984) muestra que el Efecto Día Lunes se produce durante el período de no transacción, o sea, entre el precio de cierre del día viernes y el precio de apertura del día lunes. Aggarwal y Rivoli (1989) analizaron los mercados accionarios de Asia, particularmente Hong Kong, Singapur, Malasia y Filipinas, encontrando un retorno bajo de los días lunes y también un fuerte efecto negativo para el día martes para estos mercados. La explicación que se da para el efecto martes es la correlación que existe con el mercado accionario de Nueva York. Solnik y Bousquet (1990) encontraron una significativa persistencia de retornos negativos los días martes en el mercado accionario francés. Yadav y Pope (1992) analizaron el mercado inglés y el canadiense encontrando evidencia del Efecto Día de Semana. Por su parte, Dubois y Louvel (1996) analizaron el Efecto Día de Semana para los principales mercados accionarios mundiales, concluyendo que la rentabilidad media de los lunes es negativa, mientras que la de los miércoles está sobre el promedio de la rentabilidad diaria para los mercados accionarios europeos. Berument y 
Kiymaz (2001) estudiaron el Efecto Día de Semana en retorno y volatilidad para el Índice S\&P, encontrando evidencia de bajos retornos los lunes y alta volatilidad los días viernes.

Brusa y Liu (2004) han tratado de ir más allá de la detección del efecto y exploran una explicación al Efecto Día de Semana en el mercado norteamericano, relacionando su causa a una relación positiva de los retornos y los días en que los inversores institucionales hacen sus transacciones, lo cual aumenta la actividad bursátil. Steeley (2001) encuentra evidencia que el Efecto Día de Semana ha ido desapareciendo en Inglaterra en la década de los 90.

En la última década también se han realizado estudios que buscan determinar la presencia del Efecto Día de Semana utilizando mejores técnicas de estimación, principalmente basadas en modelos GARCH. Por ejemplo, Kiymaz y Berument (2003) analizaron los mercados de Estados Unidos, Canadá, Alemania, Japón e Inglaterra encontrando anomalías en retornos y varianza en el período 19882002. Por su parte, Balaban et al. (2001) utilizaron un modelo GJR-GARCH para determinar evidencia del Efecto Día de Semana para diecinueve mercados accionarios, encontrando evidencia de anomalías de retorno en siete de ellos y anomalías de volatilidad en ocho de los mercados. Más recientemente, Charles (2010) analizó el Efecto Día de Semana para seis mercados accionarios europeos, encontrando evidencia del efecto tanto en la rentabilidad como en la volatilidad para los mercados de Atenas, París y Dublín. Para los mercados de Milán, Zurich y Helsinki solo halló evidencia del efecto en la volatilidad.

En el caso de los mercados emergentes, Ajayi et al. (2004) analizan once mercados accionarios de Europa del Este, utilizando técnicas tradicionales de regresión lineal sobre la serie de retornos y variables auxiliares para cada día. El promedio de la rentabilidad diaria de los días lunes para seis mercados fue negativo, sin embargo sólo dos de estos mercados presentan una diferencia estadísticamente significativa.

Choudhry (2000) analiza los principales mercados accionarios de Asia aplicando un modelo GARCH. En particular, estudia los mercados accionarios de Hong Kong, Indonesia, Japón, Malasia, Singapur, Taiwán y Tailandia, encontrando evidencia del Efecto Día de Semana en rentabilidad y volatilidad para el período analizado 1990-1995.

Para el mercado latinoamericano, Kristjanpoller (2009) analizó mercados accionarios de México, Brasil, Chile, Argentina, Perú y Colombia, utilizando regresiones lineales sobre la serie de retornos, así como variables auxiliares para cada día. El Efecto Lunes fue evidenciado para los mercados de Brasil, México, Chile y Argentina, mientras que el Efecto Viernes fue detectado en los mercados de Brasil, Chile, Colombia y Perú. Rivera (2009) analizó los índices bursátiles de Colombia encontrando evidencia del Efecto Día de Semana, en particular encuentra un Efecto Día Martes. Anteriormente Marshall et al. (2000) habían encontrado evidencia del Efecto Día de Semana para el mercado chileno tanto en rentabilidad como en volumen de transacción.

Tres artículos recientes particularmente influyentes son los de Sullivan et al. (2001), Baker et al. (2008) y Cho et al. (2007). Los primeros muestran que la detección de efectos calendario puede deberse a un problema de "data snooping" en el sentido de que se ha usado la misma base de datos para construir hipótesis y para probarlas, lo que genera un sesgo que habría llevado a concluir, erróneamente según los autores, que estos efectos existían. Los segundos mostraron 
que la detección del Efecto Día de Semana con modelos tipo GARCH es muy sensible a los supuestos de distribución del error. Para superar estas dificultades teóricas en la tecnología de detección, Cho et al. (2007) adoptan una aproximación no paramétrica para estudiar el Efecto Lunes para los índices Dow Jones Industrial Average (DJIA) y S\&P 500 para el período 1970-2004, Nasdaq y Russell 2000 para el período 1988-2004 y el Nikkei 225 y FTSE 100 para el período 1990-2004. El análisis lo realizan utilizando criterios de Dominancia Estocástica, encontrando evidencia del Efecto Lunes en la mayoría de los mercados en segundo y tercer orden de dominancia. El efecto es más fuerte en los índices Nasdaq y Russell 2000 que en DJIA y S\&P 500.

\section{Metodología y Datos}

Los mercados accionarios latinoamericanos analizados son Brasil, México, Chile, Colombia, Argentina y Perú, utilizándose los siguientes índices respectivamente: Bovespa, IPyC, IPSA, IGBC, Merval y ISBVL. El valor del índice del mercado $i$ en el día $t\left(I_{i, t}\right)$ corresponde a su valor de cierre en moneda doméstica para el período comprendido entre el 2 de enero de 1993 y el 31 de diciembre de 2007. Los valores fueron extraídos de la base de datos Economática.

La rentabilidad diaria del índice del mercado $i$ se calcula entonces como:

$$
R_{i, t}=\ln \left(I_{i, t} / I_{i, t-1}\right)
$$

La Tabla 1 muestra un resumen de estadísticas descriptivas para los índices utilizados. Para aislar el Efecto Día Pre Festivo, y así solamente determinar el Efecto Día de Semana, se sacan del análisis todas las semanas que contienen algún día festivo entre el lunes y el viernes, inclusive ${ }^{4}$.

De la Tabla 1 se puede observar que todos los índices analizados en el período 1993-2008 tienen una rentabilidad promedio diaria positiva en moneda doméstica. Con respecto a la desviación estándar, se puede observar que el Bovespa fue el índice con más alta desviación estándar en el período de análisis, pero a su vez es el de menor coeficiente de variación. Caso especial es el del Merval, el cual tuvo la menor rentabilidad diaria promedio, pero un coeficiente de variación que supera al resto en un orden de magnitud.

Las rentabilidades diarias del mercado colombiano (IGBC) se distribuyen simétricamente, mientras que los índices Bovespa, IPSA y ISVL tienen un sesgo hacia la derecha de la media y los índices IPyC y Merval tienen un sesgo hacia la izquierda de la media. Sobre la Kurtosis, las series de todos los índices analizados son más bien concentradas. Por último, el indicador Jarque-Bera muestra que ninguna de las distribuciones de retornos es normal y por lo tanto, en una regresión simple, se rechazaría el supuesto de normalidad sobre los errores, lo que refuerza la decisión de adoptar una aproximación no paramétrica.

4 La última fila de la Tabla 1 refleja la eliminación de algunas semanas puesto que el número de observaciones no es el mismo en los distintos países. Las semanas que permanecen en la base son entonces completas, lo que origina que en dicha fila el número de observaciones es múltiplo de cinco. 


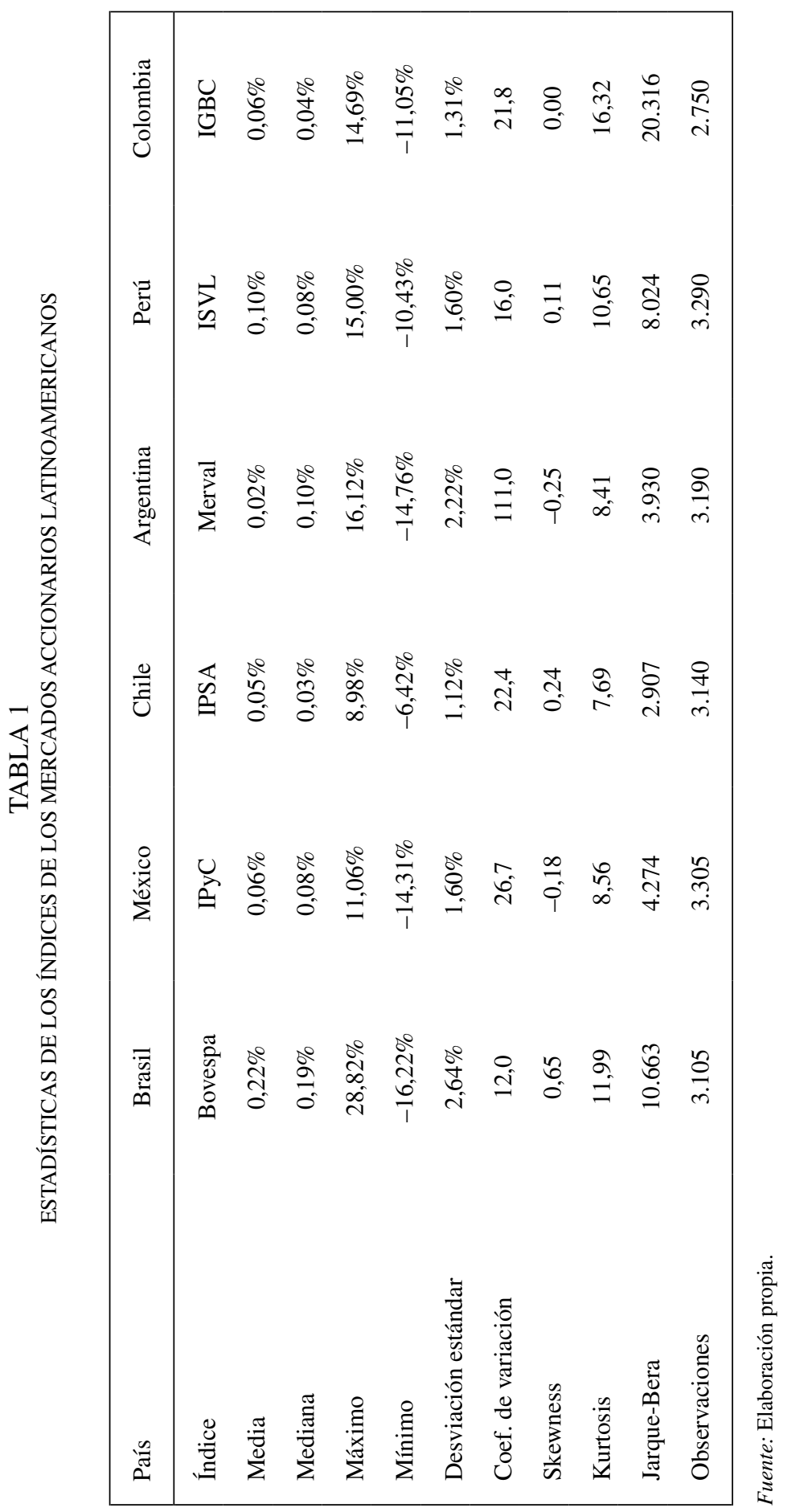


Para determinar la existencia del Efecto Día de Semana en el período de análisis se aplicará el concepto de Dominancia Estocástica. Trabajos pioneros en el desarrollo de esta herramienta son los de Hadar y Rusell (1969), Hanoch y Levy (1969) y Rothchild y Stiglitz (1970), entre otros. Sin embargo, recientemente su utilización ha tenido un nuevo impulso debido al desarrollo de herramientas estadísticas que permiten someter su detección a un test de hipótesis estándar (Linton et al., 2005; Cho et al., 2007, entre otros).

En particular para este estudio, la evidencia del Efecto Día de Semana se estudiará con la Dominancia Estocástica de Primer y Segundo Orden. La Dominancia Estocástica de Primer Orden (DEPO) es un criterio que implica la preferencia de un escenario aleatorio por sobre otro independientemente de la función de utilidad que un individuo presente, siempre que sea creciente en los niveles de riqueza. Por otra parte, la Dominancia Estocástica de Segundo Orden (DESO) es un criterio de preferencia que está restringido a funciones de utilidad de individuos aversos al riesgo. Las definiciones formales son las siguientes (Levy, 2006):

Dominancia Estocástica de Primer Orden (DEPO): La distribución $F(\cdot)$ domina estocásticamente a $G(\cdot)$ en primer orden si para toda función no decreciente $u: I R \rightarrow I R$ se tiene: $E_{F}(u(x)) \geq E_{G}(u(x))$.

Dominancia Estocástica de Segundo Orden (DESO): La distribución $F(\cdot)$ domina estocásticamente a $G(\cdot)$ en segundo orden si para toda función no decreciente y cóncava $u: I R_{+} \rightarrow I R$ se tiene: $E_{F}(u(x)) \geq E_{G}(u(x))$.

\section{Proposición $1^{5}$}

La distribución $F(\cdot)$ domina estocásticamente a $G(\cdot)$ en primer orden si y solo si $F(x) \leq G(x) \quad \forall x \in I R$.

La distribución $F(\cdot)$ domina estocásticamente a $G(\cdot)$ en segundo orden si y solo si $\int_{0}^{x}[F(t)-G(t)] d t \geq 0 \quad \forall x \geq 0$.

Existen dos formas de revisar si existe dominancia estocástica de la distribución de retornos de un día por sobre otro. La primera consiste simplemente en chequear si la condición establecida en la proposición 1 se satisface o no. Se trata de un procedimiento que arroja un resultado binario sencillo: hay o no dominancia de cada orden. El Anexo 1 contiene los resultados de la aplicación de ese procedimiento, cuyos resultados evidentemente están expuestos a la crítica de Sullivan et al. (2001). La segunda surge de considerar que la serie de datos de retorno es una realización particular de una variable aleatoria ${ }^{6}$, por lo tanto, corresponde aplicar un test estadístico de dominancia estocástica (desarrollado por Cho et al., 2007). Cabe notar que la presencia de dominancia en la primera

5 Esta proposición es ampliamente conocida en la literatura. Una posible fuente es Levy (2006).

6 De hecho, este procedimiento sigue la misma línea de Sullivan et al. (2001) al reconocer que los datos son realizaciones de variables aleatorias y se testea la presencia o ausencia de los efectos mediante técnicas de bootstraping. 
aproximación no es necesaria ni suficiente para que exista dominancia en la segunda. Por ejemplo, una distribución $F(\cdot)$ podría estar por debajo de otra distribución $G(\cdot)$ en todo su dominio salvo en un punto, por lo que en la primera aproximación no habría dominancia de primer orden de $F(\cdot)$ sobre $G(\cdot)$, pero sí podría haberla en términos estadísticos. A la inversa, una distribución $F(\cdot)$ podría estar infinitesimalmente por debajo de otra distribución $G(\cdot)$ en todo su dominio, por lo que el criterio binario diría que hay dominancia de primer orden de $F(\cdot)$ sobre $G(\cdot)$, sin embargo, bajo el segundo criterio la diferencia entre ambas podría no ser estadísticamente significativa.

La aproximación de Cho et al. (2007) consiste en la aplicación de test de hipótesis relacionados a la existencia de estas dos anomalías. En este trabajo se genera un set de diez hipótesis, de las cuales dos son generales, cuatro apuntan a la identificación del Efecto Día Lunes y las restantes cuatro se refieren al Efecto Fin de Semana. Las hipótesis nulas propuestas son:

$\mathrm{H}_{0}{ }^{1}$ : existe un día que domina a todos los demás días,

$\mathrm{H}_{0}{ }^{2}$ : existe un día que es dominado por todos los demás días,

$\mathrm{H}_{0}{ }^{3}$ : el día lunes es dominado por todos los demás días,

$\mathrm{H}_{0} 4$ : el día lunes es dominado por al menos un día,

$\mathrm{H}_{0}{ }^{5}$ : el día lunes domina a todos los demás días,

$\mathrm{H}_{0}^{6}$ : el día lunes domina al menos a un día,

$\mathrm{H}_{0}{ }^{7}$ : el día viernes es dominado por todos los demás días,

$\mathrm{H}_{0}{ }^{8}$ : el día viernes es dominado por al menos un día,

$\mathrm{H}_{0}{ }^{9}$ : el día viernes domina a todos los demás días,

$\mathrm{H}_{0}{ }^{10}$ : el día viernes domina al menos a un día.

Las primeras dos hipótesis, $\mathrm{H}_{0}{ }^{1} \mathrm{y}_{0}{ }^{2}$, son generales para evidenciar la existencia de alguna anomalía de Efecto Día, ya sea que los retornos de un día dominen a los retornos de todos los demás días o que los retornos un día sean dominados por los retornos de todos los otros días.

$\mathrm{La} \mathrm{H}_{0}{ }^{3}$ es la hipótesis clave para determinar el Efecto Día Lunes. La hipótesis $\mathrm{H}_{0}{ }^{4}$ permitiría detectar un Efecto Día Lunes parcial. Por su parte, las hipótesis $\mathrm{H}_{0}{ }^{5} \mathrm{y}_{0}{ }^{6}$ demostrarían un Efecto Lunes inverso e inverso parcial. El Efecto Fin de Semana se ve reflejado completamente en la hipótesis $\mathrm{H}_{0}{ }^{9}$ y parcialmente en la $\mathrm{H}_{0}{ }^{10}$, mientras que un Efecto Fin de Semana inverso e inverso parcial queda reflejado en las hipótesis $\mathrm{H}_{0}^{7} \mathrm{y}_{0}^{8}$.

En el análisis que sigue, cada serie de rentabilidad de un mercado específico ha sido dividida en varias series de retorno $X_{k}$ con $k=1, \ldots, 5$, siendo 1 el día lunes, 2 el martes y así sucesivamente. Cada una de las hipótesis planteadas se puede expresar como un funcional de la función de distribución de estos retornos. Sea $X$ el soporte de $X_{k}$ con $k=1, \ldots, 5$ y $s$ el orden de la dominancia estocástica. Para cada $k, l=1, \ldots, 5, s=1,2$ y $x \in X$ se define:

$$
\begin{gathered}
D_{k}^{(1)}(x)=F_{k}(x) ; \quad D_{k}^{(s)}(x)=\int_{-\infty}^{x} D_{k}^{(s-1)}(t) d t, \quad s \geq 2 ; \\
\Delta_{k, l}^{(s)}(x)=D_{k}^{(s)}(x)-D_{l}^{(s)}(x) ;
\end{gathered}
$$


En base a estas expresiones se define además:

$$
\begin{aligned}
& d_{1 s}^{*}=\min _{k} \max _{l \neq k} \sup _{x \in X} \Delta_{k, l}^{(s)}(x) ; \quad d_{2 s}^{*}=\min _{k} \max _{l \neq k} \sup _{x \in X} \Delta_{l, k}^{(s)}(x) ; \\
& d_{3 s}^{*}=\max _{k \neq 1} \sup _{x \in X} \Delta_{k, 1}^{(s)}(x) ; \quad d_{4 s}^{*}=\min _{k \neq 1} \sup _{x \in X} \Delta_{k, 1}^{(s)}(x) ; \\
& d_{5 s}^{*}=\max _{k \neq 1} \sup _{x \in X} \Delta_{1, k}^{(s)}(x) ; \quad d_{6 s}^{*}=\min _{k \neq 1} \sup _{x \in X} \Delta_{1, k}^{(s)}(x) ; \\
& d_{7 s}^{*}=\max _{k \neq 5} \sup _{x \in X} \Delta_{k, 5}^{(s)}(x) ; \quad d_{8 s}^{*}=\min _{k \neq 5} \sup _{x \in X} \Delta_{k, 5}^{(s)}(x) ; \\
& d_{9 s}^{*}=\max _{k \neq 5} \sup _{x \in X} \Delta_{5, k}^{(s)}(x) ; \quad d_{10 s}^{*}=\min _{k \neq 5} \sup _{x \in X} \Delta_{5, k}^{(s)}(x) ;
\end{aligned}
$$

Dados estos funcionales se puede establecer la hipótesis nula y su alternativa de la siguiente forma: $\mathrm{H}_{\mathrm{o}}{ }^{j}: d_{j s}{ }^{*} \leq 0$ versus $\mathrm{H}_{1}{ }^{j}: d_{j s}{ }^{*}>0$ para $j=1, \ldots, 10$.

Ahora bien, dado el carácter discreto de una muestra de retornos se hace necesario reemplazar el estadístico $d_{j s}^{*}$ por una aproximación $D j_{N}^{(s)}$ construida sobre una muestra de tamaño $N$. Por ejemplo, para la hipótesis nula $\mathrm{H}_{0}{ }^{1}$ el estadístico se define de la siguiente forma (Linton et al., 2005):

$$
D 1_{N}^{(s)}=\min _{k} \max _{l \neq k} \sup _{x \in X} \sqrt{N}\left[\bar{D}_{N}^{(s)}\left(x ; \bar{F}_{k}\right)-\bar{D}_{N}^{(s)}\left(x ; \bar{F}_{l}\right)\right]
$$

donde

$$
\bar{D}_{N}^{(s)}\left(x ; \bar{F}_{k}\right)=\frac{1}{N(s-1) !} \sum_{i=1}^{N} 1\left(X_{k i} \leq x\right)\left(x-X_{k i}\right)^{s-1} \text { para } k=1, \ldots, 5
$$

Los demás estadísticos para las restantes hipótesis son definidos de manera análoga. El rechazo de una de las hipótesis se basa en valores suficientemente grandes del estadístico, para lo que se requiere obtener un $p$-value asociado al test. La distribución asintótica del p-value se obtiene utilizando una metodología de muestreos múltiples (Linton et al., 2005) que se desarrolla de la forma siguiente. Sea $W_{N}$ el estadístico $D j_{N}^{(s)}$ para $j=1, \ldots, 10$.

(a) Se calcula el estadístico $W_{N}$ para toda la muestra completa $W_{N}=\left\{Z_{i}=\left(X_{1 i}, \ldots\right.\right.$, $\left.\left.X_{5 i}\right)^{\mathrm{T}}: i=1, \ldots, N\right\}$.

(b) Se generan submuestras $W_{N, b, i}=\left\{Z_{i}, \ldots, Z_{i+b-1}\right\}$ de tamaño $b$ para $i=1, \ldots, N-b+1$.

(c) Se calcula el estadístico $W_{N, b, i}$ para cada una de las submuestras $W_{N, b, i}$.

(d) Se aproxima el valor asintótico del $p$-value mediante:

$$
p_{s, b}=\frac{1}{N-b+1} \sum_{i=1}^{N-b+1} 1_{\left(W_{N, b, i}>W_{N}\right)}
$$


El tamaño de las submuestras depende del tamaño de la muestra total y debe satisfacer que $b \rightarrow \infty$ y $b / N \rightarrow 0$ cuando $N \rightarrow \infty$.

Si el estadístico $W_{N}$ es positivo y asociado a un $p$-value suficientemente pequeño entonces es posible rechazar la correspondiente hipótesis nula a un nivel de confianza " $p$ ".

\section{Resultados EMpíricos}

\subsection{Análisis de dominancia con muestra completa}

La evidencia encontrada a partir de una aproximación binaria, reportada en Anexo 1, podría ser validada o no a través de una aproximación estadística. Por tal razón se procedió a aplicar la batería de tests no paramétricos sugeridos por Cho et al. (2007).

En la Tabla 2 se reportan los resultados obtenidos para la dominancia de primer orden en cada una de las hipótesis establecidas. Los números achurados muestran aquellos resultados en donde es posible rechazar la hipótesis nula al $5 \%$ de significancia. Se observa evidencia estadística para ambos efectos calendario en todos los mercados. De hecho, las hipótesis $\mathrm{H}_{0}{ }^{1}, \mathrm{H}_{0}{ }^{2}, \mathrm{H}_{0}{ }^{3}$ y $\mathrm{H}_{0}^{4}$ no pueden ser rechazadas en ningún mercado, lo que es consistente con un Efecto Lunes. Por otra parte, las hipótesis asociadas a un Efecto Lunes inverso, $\mathrm{H}_{0}{ }^{5} \mathrm{y}$ $\mathrm{H}_{0}{ }^{6}$, son sistemáticamente rechazadas en los distintos mercados. ${ }^{7}$ Por otra, una situación similar ocurre con las hipótesis asociadas al Efecto Fin de Semana inverso, puesto que las hipótesis $\mathrm{H}_{0}{ }^{7} \mathrm{y}_{0}{ }^{8}$ son sistemáticamente rechazadas. La excepción es Argentina, donde un efecto Fin de Semana inverso no puede ser rechazado. Finalmente, los test directos del Efecto Fin de Semana, $\mathrm{H}_{0}{ }^{9}$ y $\mathrm{H}_{0}{ }^{10}$, muestran contundentemente que tal efecto no puede ser estadísticamente descartado en ningún país analizado.

En la Tabla 3 se reportan los resultados estadísticos asociados a la dominancia estocástica de segundo orden. Se observa nuevamente que los test directos asociados al Efecto Lunes, $\mathrm{H}_{0}{ }^{3} \mathrm{y} \mathrm{H}_{0}^{4}$, no pueden ser rechazados, mientras que aquellos asociados a un Efecto Lunes inverso, $\mathrm{H}_{0}{ }^{5} \mathrm{y} \mathrm{H}_{0}{ }^{6}$, son sistemáticamente rechazados. Una notable excepción es el mercado colombiano, donde los p-values son muy altos $\left(0,1232\right.$ y 0,2236 para $\mathrm{H}_{0}{ }^{5} \mathrm{y} \mathrm{H}_{0}{ }^{6}$, respectivamente), lo que dificulta el rechazar la nula que representa la presencia de un Efecto Lunes inverso.

Por otra parte, los test directos asociados al Efecto Fin de Semana, $\mathrm{H}_{0}{ }^{9} \mathrm{y} \mathrm{H}_{0}{ }^{10}$, muestran que la presencia de tal efecto no puede ser descartada en ningún país de la muestra, mientras que los test asociados a un Efecto Fin de Semana inverso, $\mathrm{H}_{0}{ }^{7} \mathrm{y} \mathrm{H}_{0}{ }^{8}$, son sistemáticamente rechazados. La excepción sería Argentina que es el mercado que presenta los mayores $p$-values, sin embargo, éstos son cercanos al $10 \%$, por lo que se podría rechazar un Efecto Fin de Semana inverso a este nivel de confianza en toda la muestra. En suma, las Tablas 2 y 3 muestran que las anomalías de calendario son además estadísticamente significativas, lo que confirma la presencia del fenómeno.

7 En particular, note que el mayor $p$-value para $\mathrm{H}_{0}{ }^{5}$ es de 0,1107 (Argentina), lo que indica que prácticamente en todos los mercados $\mathrm{H}_{0}{ }^{5}$ se rechazaría al $10 \%$ de confianza. 


\begin{tabular}{|c|c|c|c|c|}
\hline 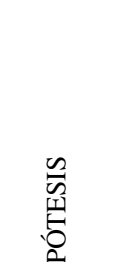 & $\begin{array}{l}\frac{\pi}{0} \\
\text { है } \\
\frac{0}{0}\end{array}$ & $\begin{array}{l}\text { U } \\
\stackrel{0}{0}\end{array}$ & 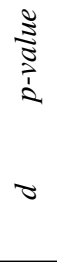 & 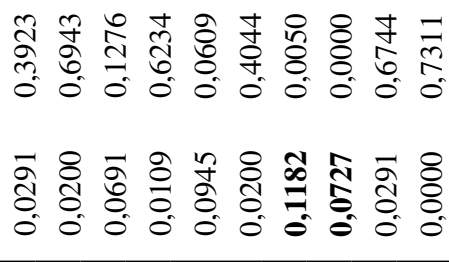 \\
\hline 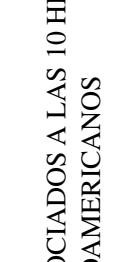 & 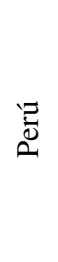 & $\stackrel{s}{\curvearrowright}$ & 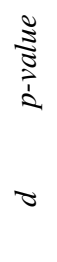 & 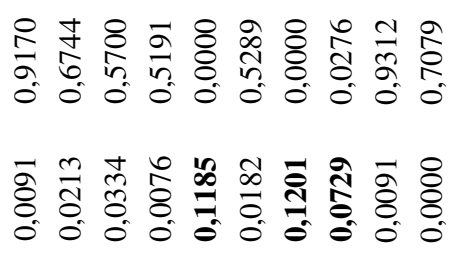 \\
\hline 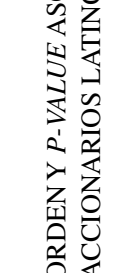 & 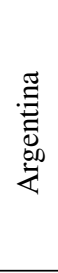 & 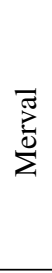 & 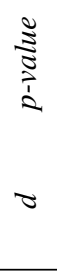 & 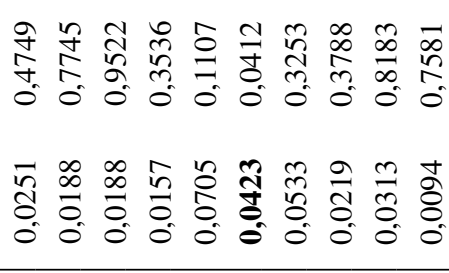 \\
\hline 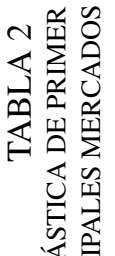 & $\stackrel{\stackrel{0}{ت}}{\stackrel{U}{U}}$ & $\begin{array}{l}\mathbb{\varkappa} \\
\Leftrightarrow\end{array}$ & 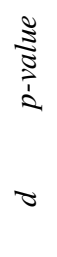 & 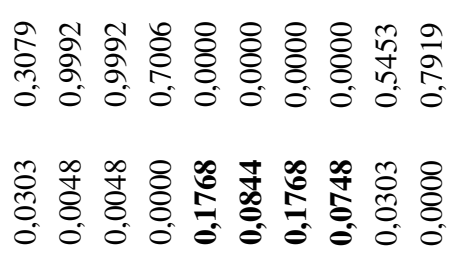 \\
\hline 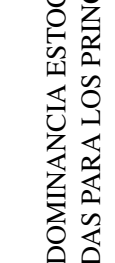 & 总 & 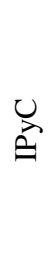 & 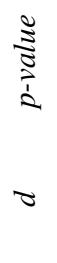 & 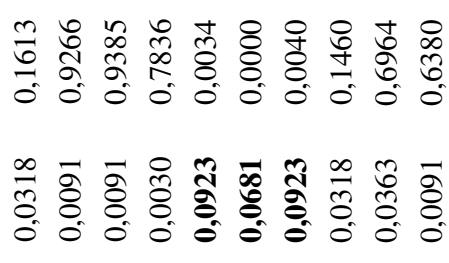 \\
\hline 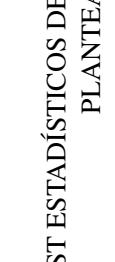 & 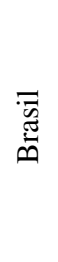 & 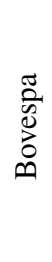 & 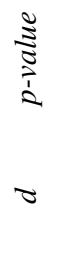 & 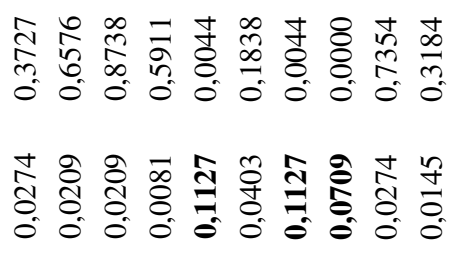 \\
\hline & 光 & 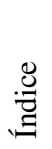 & 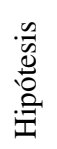 & $\neg \boxminus \boxminus \geq>5 \equiv$ \\
\hline
\end{tabular}




\begin{tabular}{|c|c|c|c|c|}
\hline 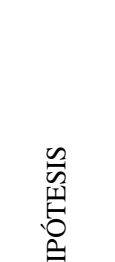 & $\begin{array}{l}. \frac{\pi}{0} \\
\text { है } \\
\frac{0}{0}\end{array}$ & 我 & 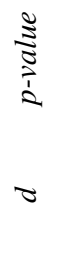 & 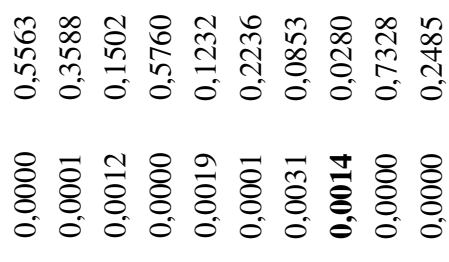 \\
\hline 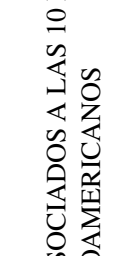 & 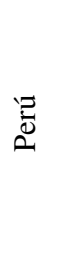 & $\stackrel{s}{\curvearrowright}$ & 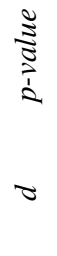 & 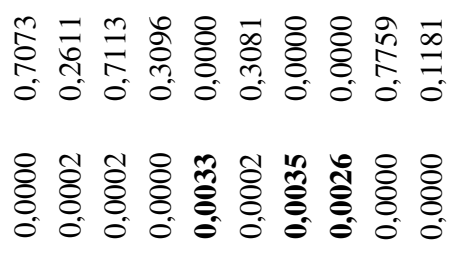 \\
\hline 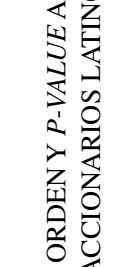 & 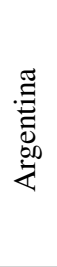 & 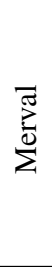 & 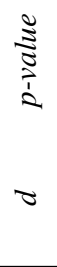 & 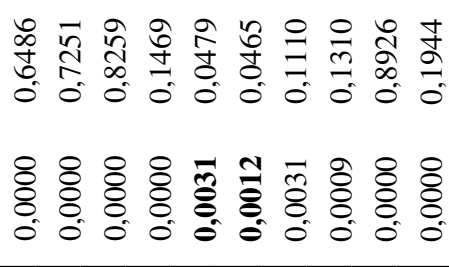 \\
\hline 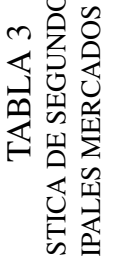 & $\frac{\mathscr{Z}}{己}$ & $\underset{\mathscr{E}}{\mathbb{E}}$ & 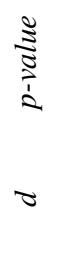 & 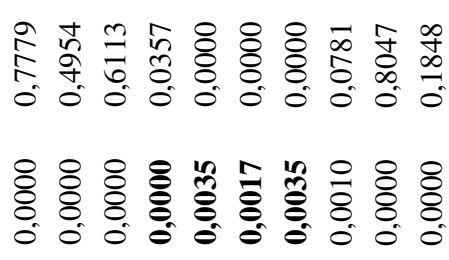 \\
\hline 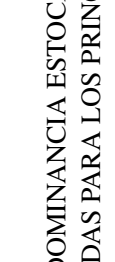 & 递 & 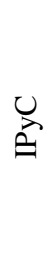 & 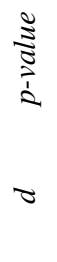 & 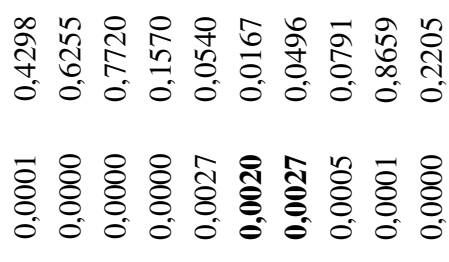 \\
\hline 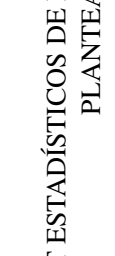 & 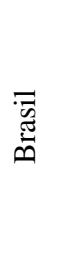 & $\begin{array}{l}\frac{\pi}{2} \\
00 \\
0 \\
0 \\
0\end{array}$ & 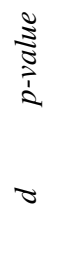 & 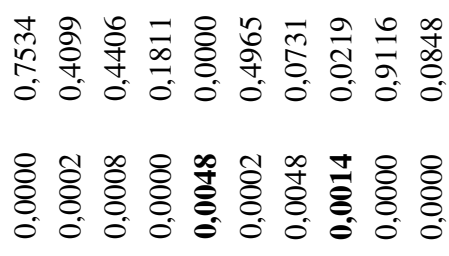 \\
\hline & 光 & 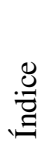 & 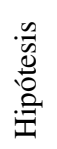 & $\neg \boxminus \boxminus \geq>5 \equiv$ \\
\hline
\end{tabular}




\subsection{Análisis de dominancia con submuestras}

El mismo procedimiento de la sección anterior se aplicó por separado a dos submuestras, la primera para el período 1993-2000 y la segunda en el período 2001-2007. Las Tablas 4 y 5 muestran los resultados de los tests de dominancia de primer orden en el primer y segundo período, respectivamente. Análogamente, las Tablas 6 y 7 muestran los resultados de los test de dominancia de segundo orden.

Los resultados obtenidos con este análisis son en su gran mayoría consistentes entre los dos períodos y confirman además los resultados obtenidos en el análisis del período total. En particular, las hipótesis $\mathrm{H}_{0}{ }^{3} \mathrm{y} \mathrm{H}_{0}{ }^{4}$ no pueden ser rechazadas en ningún mercado en primer orden, lo que es consistente con un Efecto Lunes. Por su parte, los test directos del Efecto Fin de Semana, $\mathrm{H}_{0}{ }^{9}$ y $\mathrm{H}_{0}{ }^{10}$, muestran contundentemente que tal efecto no puede ser estadísticamente descartado en primer orden en ningún país analizado.

Para el caso de dominancia de segundo orden, se mantienen los resultados de las hipótesis $\mathrm{H}_{0}{ }^{3} \mathrm{y} \mathrm{H}_{0}{ }^{4}$ no pudiendo ser rechazadas en ningún mercado en segundo orden, lo que es consistente con un Efecto Lunes, excepto para el caso de Chile y Perú solo en el primer período de análisis. Por su parte, los test directos del Efecto Fin de Semana, $\mathrm{H}_{0}{ }^{9}$ y $\mathrm{H}_{0}{ }^{10}$, muestran que tal efecto no puede ser estadísticamente descartado en los países estudiados, excepto para el caso de Perú en el primer período y Colombia y Brasil para el segundo período.

En suma, al dividir el período en dos no se encontró evidencia de que las anomalías de calendario hayan tendido a desaparecer en los mercados latinoamericanos, por el contrario, se detectó su persistencia en el tiempo.

\subsection{Significancia económica de las anomalías}

En esta sección nos concentraremos en cuantificar la importancia económica de estas anomalías a través de un simple ejercicio. Se compararon dos estrategias alternativas:

i. Invertir al final de los días lunes en los índices y luego vender al final del día viernes.

ii. Mantener una estrategia de largo plazo de buy and hold en el índice.

Se determinó la máxima comisión variable que se podría pagar para sacar ventaja de la presencia de la anomalía. Este análisis se realizó tanto para el período completo como para los dos subperíodos definidos. La Tabla 8 resume los resultados.

Se puede observar que para el caso de todo el período para los mercados accionarios de Argentina, Chile y México que con una comisión menor al 0,07\% hubiera sido más rentable la estrategia de invertir al final de los días lunes en los índices y luego vender al final del día viernes que haber tenido una estrategia tradicional de buy and hold de largo plazo en el índice. Para los mercados accionarios de Brasil, Perú y Colombia el ejercicio no es aplicable (N/A) en el período completo, ya que si bien la rentabilidad de los días lunes es menor que la de los otros días, de todas formas es positiva, lo cual no deja cabida a la estrategia activa de comprar los lunes y vender los viernes.

Interesante es observar que para el segundo período de análisis, 2001-2007, en todos los mercados analizados excepto el mercado peruano se puede implementar una estrategia activa rentable, siempre cuando existan comisiones menores a las calculadas. 


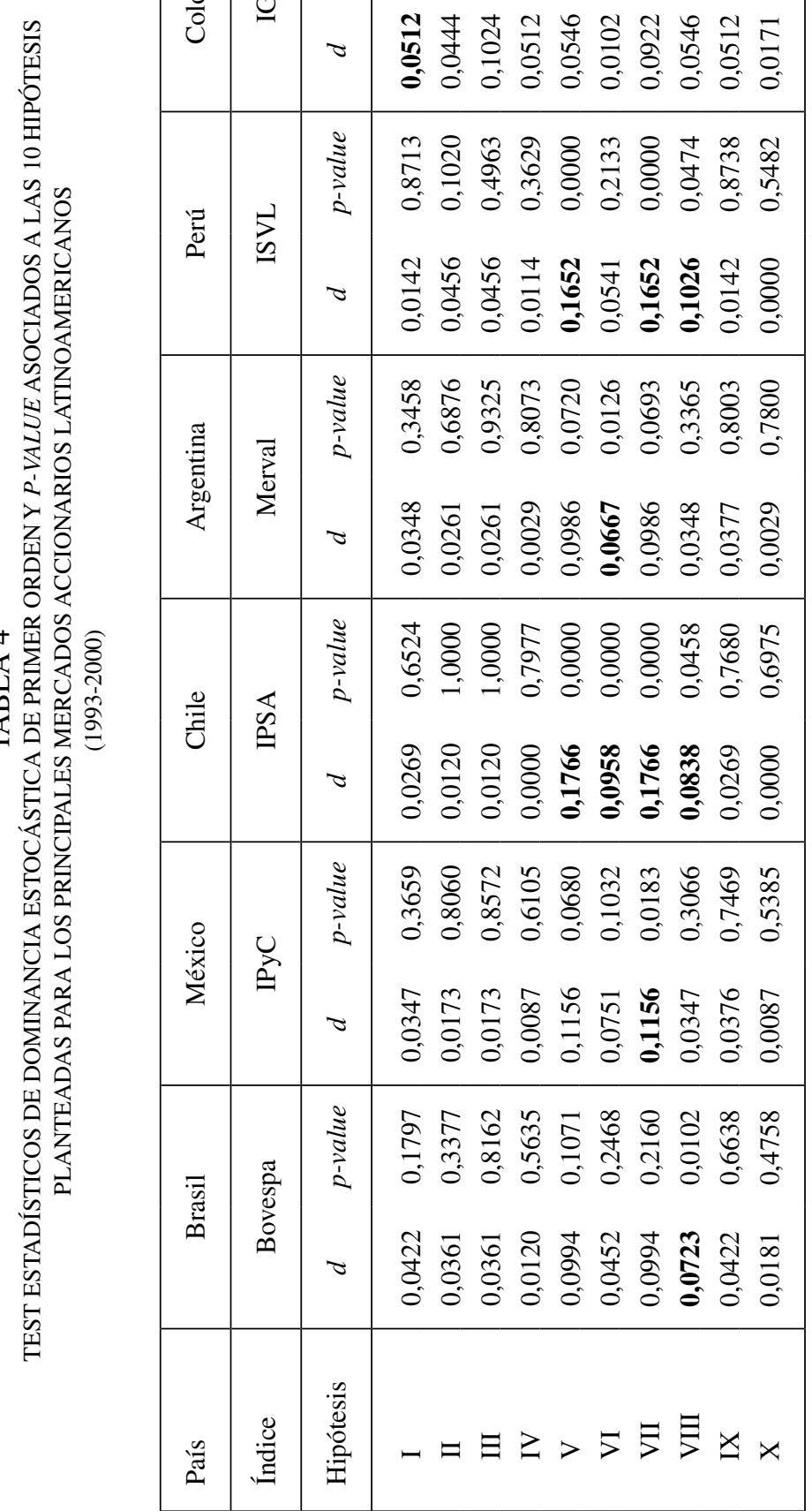




\begin{tabular}{|c|c|c|c|c|c|c|c|c|c|c|c|c|}
\hline \begin{tabular}{l}
$n$ \\
\multicolumn{2}{c}{} \\
II \\
10 \\
0
\end{tabular} & ن & & $\tau$ & 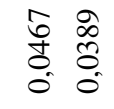 & $\begin{array}{l}\stackrel{\infty}{\hat{s}} \\
0 \\
0\end{array}$ & हे & $\frac{3}{a}$ & 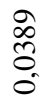 & $\frac{3}{2}$ & مै & & \\
\hline 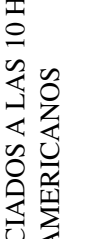 & 20 & $\stackrel{3}{\Omega}$ & 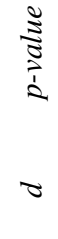 & 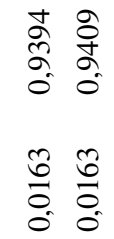 & $\begin{array}{l}\hat{n} \\
\tilde{n} \\
0 \\
0 \\
\hat{0} \\
0\end{array}$ & 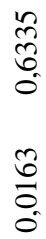 & $\begin{array}{l}\stackrel{\infty}{ \pm} \\
\vdots \\
0 \\
n \\
0 \\
0 \\
0\end{array}$ & $\begin{array}{l}2 \\
\sigma \\
0 \\
0 \\
0 \\
0 \\
0 \\
0\end{array}$ & $\begin{array}{l}8 \\
8 \\
8 \\
0 \\
\text { ह1 } \\
\frac{1}{0}\end{array}$ & $\frac{\hat{N}}{0}$ & & \\
\hline 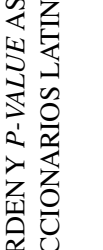 & 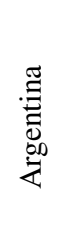 & 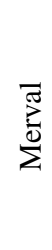 & 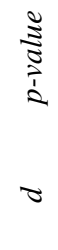 & 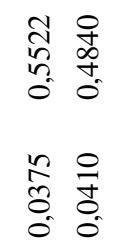 & $\begin{array}{l}n \\
0 \\
+ \\
0 \\
0 \\
0 \\
0 \\
0\end{array}$ & 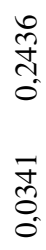 & $\begin{array}{l}0 \\
+0 \\
\frac{1}{0} \\
8 \\
8 \\
8 \\
0\end{array}$ & 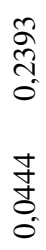 & $\begin{array}{l}\frac{m}{+} \\
\frac{\infty}{0} \\
\frac{0}{0} \\
0 \\
0\end{array}$ & 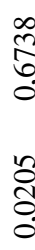 & & \\
\hline 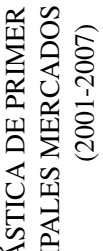 & $\frac{\mathscr{Z}}{\bar{\theta}}$ & 芯 & 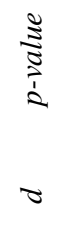 & $\begin{array}{ll}8 & \overline{8} \\
8 & \circ \\
0 & 0 \\
0 & 0 \\
0 & \infty \\
0 & 8 \\
0 & 0 \\
0 & 0\end{array}$ & $\begin{array}{l}\bar{\infty} \\
\delta \\
0 \\
0 \\
\infty \\
\delta \\
\delta \\
0\end{array}$ & $\begin{array}{l}4 \\
2 \\
\infty \\
2 \\
0 \\
0 \\
8 \\
8 \\
8 \\
0\end{array}$ & $\begin{array}{l}8 \\
8 \\
0 \\
0 \\
\frac{1}{0} \\
0\end{array}$ & 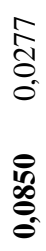 & $\begin{array}{l}8 \\
8 \\
0 \\
0 \\
10 \\
\frac{0}{0}\end{array}$ & $\stackrel{e}{\circ}$ & & \\
\hline 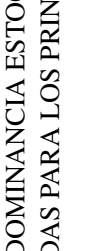 & 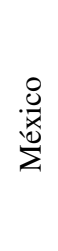 & 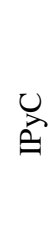 & 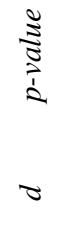 & 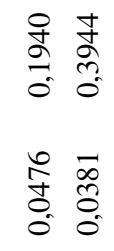 & \begin{tabular}{l}
8 \\
\multirow{2}{+}{} \\
0 \\
0 \\
$\infty$ \\
0 \\
0 \\
0
\end{tabular} & $\begin{array}{l}\bar{\sigma} \\
0 \\
0 \\
\hat{0} \\
0 \\
0\end{array}$ & $\begin{array}{l}2 \\
\text { ले } \\
0 \\
0 \\
0 \\
0 \\
0 \\
0\end{array}$ & $\begin{array}{c}0 \\
\frac{0}{0} \\
0 \\
10 \\
0 \\
0 \\
0\end{array}$ & $\begin{array}{l}1 \\
0 \\
0 \\
0 \\
\dot{0} \\
\text { + } \\
8 \\
0 \\
0\end{array}$ & $\frac{\text { g }}{0}$ & & \\
\hline 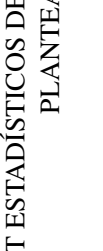 & $\begin{array}{l}\overline{0} \\
\overline{7} \\
\overline{0}\end{array}$ & $\begin{array}{l}\text { क्र } \\
\text { o } \\
\text { Dे } \\
\infty\end{array}$ & 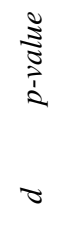 & 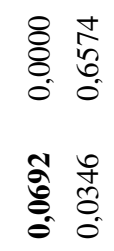 & $\begin{array}{l}\hat{0} \\
\infty \\
0 \\
0 \\
0 \\
0 \\
0 \\
0\end{array}$ & $\begin{array}{l}\infty \\
0 \\
0 \\
0 \\
0 \\
0 \\
0 \\
0\end{array}$ & $\begin{array}{l}2 \\
\text { సे } \\
0 \\
0 \\
\tilde{n} \\
\stackrel{0}{0} \\
0\end{array}$ & 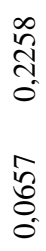 & 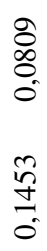 & 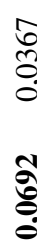 & & \\
\hline & 气 & 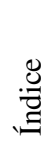 & $\begin{array}{l}\stackrel{n}{0} \\
0 \\
0 \\
0 \\
0\end{array}$ & $ー \models$ & 目 & $\geq$ & $>$ & 5 & 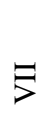 & $>$ & & \\
\hline
\end{tabular}




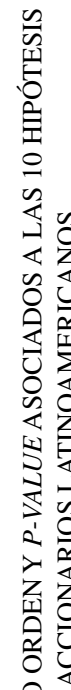

\begin{tabular}{|c|c|c|c|c|}
\hline 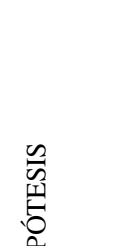 & $\begin{array}{l}\frac{. \pi}{0} \\
\frac{0}{0} \\
0\end{array}$ & $\begin{array}{l}\text { U } \\
\text { OU }\end{array}$ & 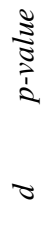 & 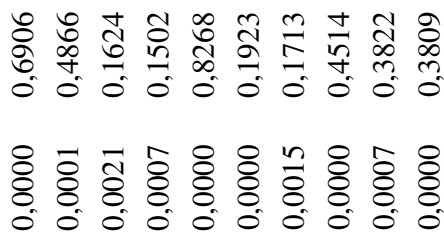 \\
\hline 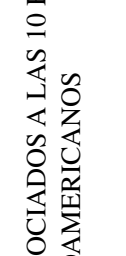 & 苛 & $\stackrel{2}{2}$ & 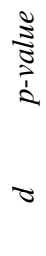 & 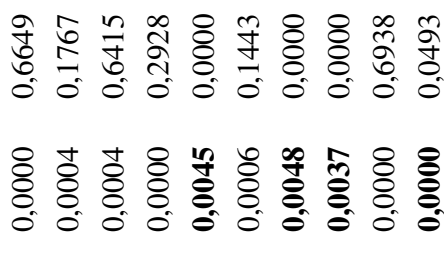 \\
\hline 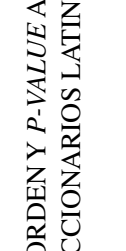 & 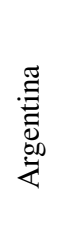 & 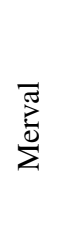 & $\begin{array}{l}\frac{0}{3} \\
\frac{\pi}{2} \\
\frac{1}{2}\end{array}$ & 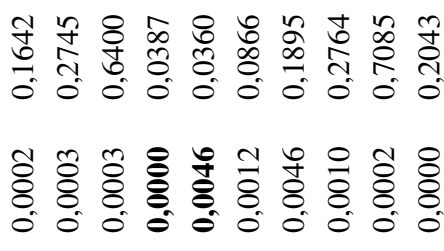 \\
\hline 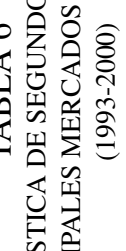 & 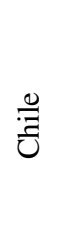 & 岕 & 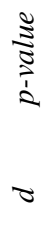 & 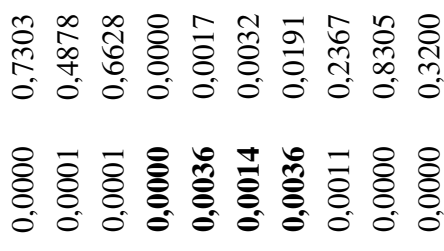 \\
\hline 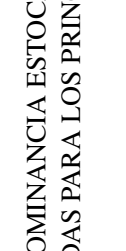 & 总 & 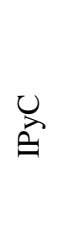 & 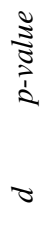 & 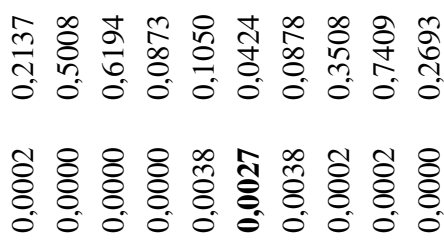 \\
\hline 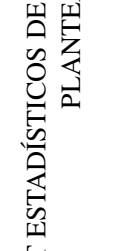 & $\begin{array}{l}\overline{\bar{a}} \\
\overline{\tilde{m}} \\
\bar{n}\end{array}$ & 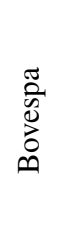 & 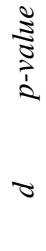 & 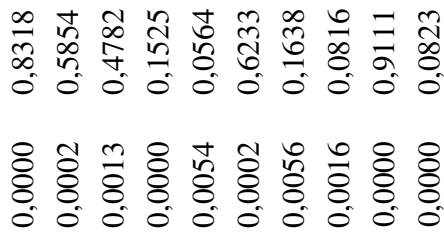 \\
\hline & 瓷 & : & 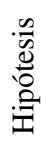 & 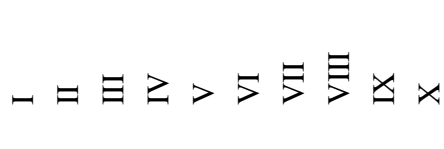 \\
\hline
\end{tabular}




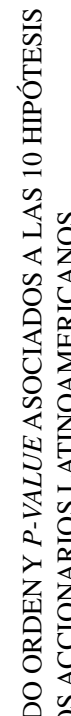

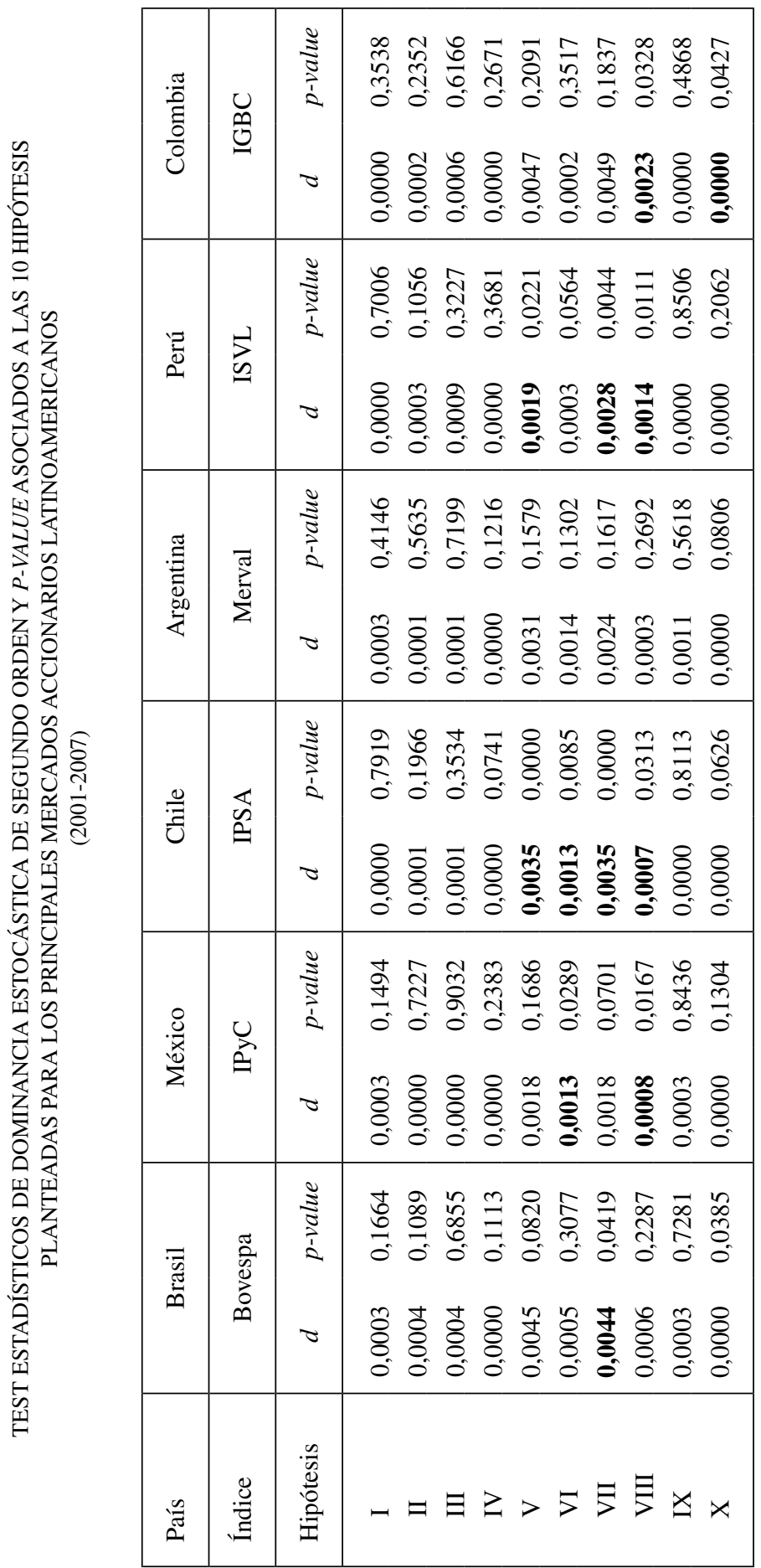


TABLA 8

COMISIÓN VARIABLE MÁXIMA A LAS TRANSACCIONES PARA INVERSIONISTAS

\begin{tabular}{|l|ccc|}
\hline \multirow{2}{*}{ País } & \multicolumn{3}{|c|}{ Período } \\
\cline { 2 - 4 } & $1993-2007$ & $1993-2000$ & $2001-2007$ \\
\hline Brasil & N/A & N/A & $0,075 \%$ \\
Argentina & $0,073 \%$ & $0,108 \%$ & $0,032 \%$ \\
Chile & $0,077 \%$ & $0,077 \%$ & $0,078 \%$ \\
Perú & N/A & $0,023 \%$ & N/A \\
Colombia & N/A & N/A & $0,045 \%$ \\
México & $0,072 \%$ & $0,128 \%$ & $0,010 \%$ \\
\hline
\end{tabular}

\section{Conclusiones}

En este artículo se estudió la presencia de anomalías de calendario en los principales mercados accionarios latinoamericanos en el período comprendido entre 1993 y 2007. El tema es particularmente controversial puesto que ha surgido evidencia de que el efecto en algunos países desarrollados ha tendido a desaparecer (Steeley, 2001), así como críticas a las tecnologías mismas de detección que han sido utilizadas en el pasado, las que podrían haber llevado a detectar un fenómeno que en realidad no existía (Sullivan et al., 2001; Baker et al., 2008).

Para estudiar la presencia o ausencia de estas anomalías se adoptó la aproximación recientemente desarrollada por Linton et al. (2005) y Cho et al. (2007), la que es robusta a las críticas metodológicas anteriormente planteadas. Se trata de una aproximación no paramétrica consistente en la detección de dominancia estocástica de la distribución de retornos de unos días por sobre otros. Las técnicas tradicionales de detección de dominancia llevan a una conclusión binaria: existe o no dominancia de cada orden. Sin embargo, los trabajos citados han hecho posible testear estadísticamente la presencia de dominancia de cualquier orden.

Los resultados muestran que las anomalías de calendario "Efecto Lunes" y "Efecto Fin de Semana" no solo siguen siendo detectadas en las principales bolsas latinoamericanas, sino que además este resultado es robusto a una partición de la muestra en los períodos 1993-2000 y 2001-2007. Otras anomalías detectadas en trabajos anteriores, sin embargo, no pasaron el test de significancia estadística. Tal es el caso, por ejemplo, del efecto martes reportado por otros autores para el índice IGBC de la bolsa colombiana. ${ }^{8}$

Se demuestra además que en varias de las bolsas estudiadas es posible identificar períodos en donde las anomalías pueden ser explotadas a través de una estrategia de inversión. Sin embargo, la imposibilidad de explotar la anomalía no puede ser interpretada como la inexistencia de ésta. En otras palabras, la presencia de la anomalía es una condición necesaria, mas no suficiente, para que exista una estrategia que permita explotarla.

Finalmente, los resultados no entregan información respecto al origen de estos fenómenos, por lo que sus causas se mantienen como un tema de investigación plenamente vigente.

$8 \quad$ Ver por ejemplo Rivera (2009). 


\section{ANEXO 1}

\section{Aproximación Binaria}

Los resultados obtenidos al aplicar un análisis binario de Dominancia Estocástica de primer orden a cada uno de los mercados accionarios analizados durante todo el período de análisis se pueden observar en la Tabla A1. Se encontró evidencia de dominancia estocástica de primer orden de la distribución de retornos del día viernes sobre otros días en algunos mercados.

El mercado accionario chileno presenta dos días dominados en primer orden por el día viernes, el día lunes y el día jueves. Por su parte, el mercado accionario colombiano presenta la anomalía que el día viernes domina al día martes en primer orden, mientras que el mercado accionario peruano evidencia la dominancia de primer orden de los retornos del día viernes sobre el día miércoles. Los mercados accionarios brasileño, mexicano y argentino no presentan dominancia de un día sobre otro en primer orden.

TABLA A1

RESULTADOS DE DOMINANCIA ESTOCÁSTICA DE PRIMER ORDEN DE LOS RETORNOS DIARIOS PARA LOS PRINCIPALES MERCADOS ACCIONARIOS LATINOAMERICANOS

\begin{tabular}{|l|cccccc|}
\hline \multirow{2}{*}{ Condición } & Brasil & México & Chile & Argentina & Perú & Colombia \\
\cline { 2 - 7 } & Bovespa & IPyC & IPSA & Merval & ISVL & IGBC \\
\hline $\begin{array}{l}\text { Viernes domina } \\
\text { a Lunes }\end{array}$ & & & Sí & & & \\
Viernes domina & & & & & Sí \\
a Martes & & & & & \\
Viernes domina & & & & & \\
a Miércoles & & & & & & \\
Viernes domina & & & & & & \\
a Jueves & & & & & & \\
\hline
\end{tabular}

Los resultados obtenidos al aplicar el análisis de Dominancia Estocástica de segundo orden a cada uno de los mercados accionarios analizados durante todo el período de análisis se pueden observar en la Tabla A2. Si se asume que los inversores son en general aversos al riesgo, la dominancia estocástica de segundo orden, a pesar de ser más débil que la de primer orden, es una razón suficiente para que un inversionista prefiera un día por sobre otro. 
TABLA A2

RESULTADOS DE DOMINANCIA ESTOCÁSTICA DE SEGUNDO ORDEN

DE LOS RETORNOS DIARIOS PARA LOS PRINCIPALES MERCADOS

ACCIONARIOS LATINOAMERICANOS

\begin{tabular}{|c|c|c|c|c|c|c|}
\hline País & Brasil & México & Chile & Argentina & Perú & Colombia \\
\hline Índice & Bovespa & IPyC & IPSA & Merval & ISVL & IGBC \\
\hline $\begin{array}{l}\text { Martes domina } \\
\text { a Lunes } \\
\text { Martes domina } \\
\text { a Miércoles }\end{array}$ & Sí & Sí & & Sí & & \\
\hline $\begin{array}{l}\text { Martes domina } \\
\text { a Jueves }\end{array}$ & Sí & & & Sí & & \\
\hline $\begin{array}{l}\text { Miércoles domina } \\
\text { a Lunes }\end{array}$ & Sí & Sí & & Sí & Sí & \\
\hline $\begin{array}{l}\text { Miércoles domina } \\
\text { a Martes }\end{array}$ & & & & & Sí & Sí \\
\hline $\begin{array}{l}\text { Miércoles domina } \\
\text { a Jueves }\end{array}$ & & Sí & Sí & Sí & & \\
\hline $\begin{array}{l}\text { Jueves domina } \\
\text { a Lunes }\end{array}$ & & Sí & & Sí & Sí & \\
\hline $\begin{array}{l}\text { Jueves domina } \\
\text { a Martes }\end{array}$ & & & & & Sí & \\
\hline $\begin{array}{l}\text { Viernes domina } \\
\text { a Lunes }\end{array}$ & Sí & Sí & Sí & Sí & Sí & Sí \\
\hline $\begin{array}{l}\text { Viernes domina } \\
\text { a Martes }\end{array}$ & Sí & & Sí & Sí & Sí & Sí \\
\hline $\begin{array}{l}\text { Viernes domina } \\
\text { a Miércoles }\end{array}$ & Sí & & Sí & Sí & Sí & Sí \\
\hline $\begin{array}{l}\text { Viernes domina } \\
\text { a Jueves }\end{array}$ & Sí & Sí & Sí & Sí & Sí & Sí \\
\hline
\end{tabular}

Al observar los resultados de la aplicación del criterio de dominancia estocástica en segundo orden se puede concluir que en los mercados analizados, con excepción del mercado accionario mexicano, la distribución de retornos del día viernes domina en segundo orden a la distribución de retornos de todos los otros días de la semana, generando evidencia del efecto de fin de semana. Incluso en el caso de México, se observa que hay dominancia de segundo orden de los retornos del viernes sobre los de lunes y jueves.

Para analizar el Efecto Día Lunes se debe observar los días que dominan los retornos del día lunes. En el caso del mercado accionario mexicano y argentino los retornos de los días martes, miércoles, jueves y viernes dominan en segundo orden a los retornos del día lunes, evidenciando el Efecto Lunes. En 
el mercado accionario brasileño solo los retornos del día jueves no dominan estocásticamente en segundo orden a los retornos del día lunes, mientras que en el mercado accionario peruano solo los retornos del día martes no dominan estocásticamente en segundo orden a los retornos del día lunes.

Para el caso de los mercados accionarios de Chile y de Colombia los retornos del día lunes son solamente dominados en segundo orden por los retornos del día viernes. En particular se puede observar que existen otras anomalías como en los mercados accionarios argentino, chileno y mexicano donde los retornos del día miércoles dominan en segundo orden a los retornos del martes. En el mercado accionario brasileño y argentino los retornos del día martes dominan a los retornos del día jueves, mientras que en los mercados accionarios de Colombia y Perú se encuentra evidencia que los retornos del día miércoles dominan a los del día martes.

\section{Bibliografía}

Aggarwal, R. and P. Rivoli (1989). "Seasonal and Day-of-the-Week Effects in Four Emerging Stock Markets”, Financial Review, 24, 541-550.

Ajayi, R. A., S. Mehdian y M. J. Perry (2004). "The Day-of-the-Week Effect in Stock Returns. Further Evidence from Eastern European Emerging Markets", Emerging Markets Finance and Trade, 40 (4), 53-62.

Baker, K., A. Rahman y S. Saadi (2008). "The Day-of-the-Week Effect and Conditional Volatility: Sensitivity of Error Distributional Assumptions", Review of Financial Economics, 17 (4), 280-295.

Balaban, E., A. Bayar y O. Z. Kan (2001). "Stock Returns, Seasonality and Asymmetric Conditional Volatility in World Equity Markets", Applied Economics Letters, 8, 263-268.

Berument H. and H. Kiymaz (2001). "The Day of the Week Effect On Stock Market Volatility", Journal of Economics and Finance, 25, 181-193.

Brusa, J. y P. Liu (2004). "The Day-of-the-Week and the Week-of-the-Month Effects: An Analysis of Investors' Trading Activities", Review of Quantitative Finance and Accounting, 23, 19-30.

Charles, A. (2010). "Does the Day-of-the-Week Effect on Volatility Improve the Volatility Forecasts?", Applied Economics Letters, 17 (3), 257-262.

Cho, Y-H., O. Linton y Y. J. Whang (2007). "Are there Monday Effects in Stock Returns: A Stochastic Dominance Approach", Journal of Empirical Finance, 14 (5), 736-755.

Choudhry, T. (2000). "Day-of-the-Week Effect in Emerging Asian Stock Markets: Evidence from the GARCH Model", Applied Financial Economics, 10, 235-242.

Cross, F. (1973). "The Behavior of Stock Prices on Fridays and Mondays", Financial Analyst Journal, 29, 67-69.

Dubois, M. y P. Louvet (1996). "The Day-of-the-Week Effect: The International Evidence", Journal of Banking and Finance, 20 (9), 1463-1484.

Fama, E. (1965). "The Behavior of Stock Market Prices", Journal of Business 38 (1), 34-105.

Fields, M. (1931). "Stock Prices: A Problem in Verification", Journal of Business, 4, 415-418. 
French, K. (1980). "Stock Returns and the Weekend Effect", Journal of Financial Economics 13 (1), 55-69.

Gibbons, R. y P. Hess (1981). "Day-of-the-Week Effects and Asset Returns", Journal of Business 54 (4), 579-596.

Hadar, J. y W. Rusell (1969). "Rules for Ordering Uncertain Prospects", American Economic Review, 59, 25-34.

Hanoch, G. y H. Levy (1969). “The Efficiency Analysis of Choices Involving Risk", Review of Economic Studies, 36, 335-346.

Kiymaz, H. y H. Berument (2003). "The Day of the Week Effect on Stock Market Volatility and Volume: International Evidence", Review of Financial Economics 12 (4), 363-380.

Kristjanpoller, W. (2009). "An Analysis of the Day-of-the-Week Effect in Latin American Stock Markets", Lecturas de Economía, 71, 189-208.

Lakonishok, J., and M. Levi (1982). "Weekend Effects on Stock Returns: A Note", Journal of Finance, 37 (3), 883-889.

Levy, H. (2006). Stochastic Dominance. Investment Decision Making under Uncertainty, Springer, second edition.

Linton, O., Maasoumi, E. y Wang Y. (2005). "Consistent Testing for Stochastic Dominance under General Sampling Schemes", Review of Economics Studies, 72, 735-765.

Marshall, P. y Walker, E. (2000). "Day of the week and size effects in emerging markets: Evidence from Chile", Revista de Análisis Económico, Vol. 15, $\mathrm{N}^{\mathrm{o}} 2,89-108$.

Rivera, D. (2009). "Modelación del Efecto del Día de Semana para los Índices Accionarios de Colombia mediante un modelo Star Garch", Revista de Economía del Rosario, 12 (1), 1-24.

Rogalski, R. (1984). "New Findings Regarding Day-of-the-Week Returns over Trading and Non-trading Periods: A Note", The Journal of Finance, 39, 1603-1614.

Rothschild, M. y J. Stiglitz (1970). “Increasing Risk: I. A Definition”, Journal of Economic Theory, 2, 225-243.

Solnik, B. y L. Bousquet (1990). "Day-of-the-Week Effect on the Paris Bourse", Journal of Banking and Finance, 14, 461-468.

Steeley, J. (2001). "A Note on Information Seasonality and the Disappearance of the Weekend Effect in the UK Stock Market", Journal of Banking and Finance, 25 (10), 1941-1956.

Sullivan, R., A. Timmermann y H. White (2001). "Dangers of Data Mining: The Case of Calendar Effects in Stock Returns", Journal of Econometrics, 105, 249-286.

Yadav P y P. Pope (1992). "Intraweek and Intraday Seasonalities in Stock Market Risk Premia: Cash v/s Futures", Journal of Banking and Finance, 16 (1), 233-270. 